\title{
Application and Development of Digital Enhancement of Traditional Sculpture Art
}

\author{
Zeyin Yang (10 \\ Hefei Normal University, Hefei, Anhui 230601, China \\ Correspondence should be addressed to Zeyin Yang; zeyin_yang0312@126.com
}

Received 21 December 2021; Revised 4 January 2022; Accepted 5 January 2022; Published 3 February 2022

Academic Editor: Tongguang $\mathrm{Ni}$

Copyright ( $) 2022$ Zeyin Yang. This is an open access article distributed under the Creative Commons Attribution License, which permits unrestricted use, distribution, and reproduction in any medium, provided the original work is properly cited.

\begin{abstract}
Sculpture art, as an important carrier of spiritual civilization, also portrays a prosperous scene as an industry with urban and cultural development. Three-dimensional technology offers a new platform for sculpture creation, allowing for the digitization of sculpture works via electronic information technology, and the display of sculpture works in front of people via displays, facilitating the exchange and dissemination of information and promoting the growth and progress of the entire sculpture creation industry. We plan to use digital enhancement technology to conduct small-scale creation experiments on traditional sculpture works, discuss the method of GA (Genetic Algorithm) in image restoration processing, investigate the method of image segmentation processing based on the genetic algorithm, and propose the method of image segmentation processing based on the fuzzy membership surface genetic algorithm, in order to verify and solve the creation difficulties of traditional sculpture works.
\end{abstract}

\section{Introduction}

As time passes, new technological means emerge one after the other. The use of new technology has resulted in significant changes in the way artists create art. The use of digital technology in the design and production of sculptures is becoming increasingly common [1]. Sculptors are increasingly turning to computers to create virtual sculptures. Sculptors can not only design sculptures with computer software but they can also simulate the space environment in which the sculptures will be displayed for a better display effect $[2,3]$. The creative process of sculpture art and even the sculpture itself have separated from the limitations of material materials thanks to three-dimensional modeling design, which expands the creative means of sculpture to the computer's three-dimensional virtual space.

Digital sculpture is a small branch of digital art. A digital sculpture is a combination of three-dimensional modeling technology and traditional easel carving art, and artistic creation by computer [4]. Digital sculpture, as a new discipline integrating art and science, has broad development space and application fields. Three-dimensional technology comes from life, develops in life, and feeds back into life.
Three-dimensional digital technology refers to a computerrelated science and technology, which identifies and processes images, sounds, characters, images, etc., through advanced cushion equipment, converts them into binary numbers "0" and " 1 " that can be identified and processed by computers, and carries out operations, processing, storage, transmission, and restoration through a series of algorithms $[5,6]$. Sculpture, as a traditional art category, plays an important role in human history. As an important cultural carrier, sculpture art has always had extraordinary significance in human social and cultural life. It is for this reason that the combination of contemporary sculpture art and the most advanced digital technology can be regarded as the inevitable trend of the times and the inevitable result of the development law of sculpture art [7]. With the support of digital technology, the creation accuracy of sculpture works will be significantly improved, and the creation cycle and cost will also be reduced, saving artists a lot of time and energy to invest in more artistic creation.

It has been more than ten years since virtual technology was used in sculptures. It has progressed from a supporting technical tool to a singular artistic expression in the hands of artists [8]. After a long period of development and 
improvement, 3D technology [9] now has a powerful data simulation capability, allowing it to simulate and display various detailed aspects in a virtual environment, such as light and shadow, object material and color, motion track and way, and geographic location, through practical operations. In this paper, digital technology is combined with traditional sculpture art's creative methods, and the research of a sculpture art creation method based on digital technology is presented in order to meet the demand for increased accuracy and efficiency in sculpture art creation.

\section{Related Work}

Although digital technology has been introduced into the process of sculpture art creation by some artists, most of the known research studies and attempts at present are the two combinations between 3D scanning technology and 3D printing technology, or digital carving technology and 3D printing technology, but it is rare to study the comprehensive application of the three technologies [10]. 3D printing technology, as the key technology of digital technology to finally create entities, has a great influence on the realization of the idea of digitalization of sculpture art. Literature $[11,12]$ is eager to use digital technology in their own artistic creations, and they regularly hold personal digital work exhibitions to demonstrate the benefits of science and technology to art and to encourage young artists to pursue careers in this field. Literature [13] investigates the characteristics and connotations of art under the influence of numbers, with the help of calculations. The majority of studies in literature [14] are focused on digital media, virtual art, and digital aesthetics, with only a few theoretical studies on the use of digital technology in sculpture. On the one hand, only sculptures on yellow mud, Chinese painting on rice papers, and prints on copper plates, according to literature [15], can be considered true works of art. Computer graphics, on the other hand, are highly technical, untouchable, and emotionless, and thus cannot be called art. Literature [16] predicts that computers will evolve from rational technology to artistic beauty and that digital technology will have limitless applications.

Augmented reality technology is a technology that "seamlessly" superimposes information from the virtual world to the real world. By superimposing information generated by computers into the real world, users can achieve sensory experiences beyond reality. In Literature [17], using an unsharp mask to enhance images, the contribution of sharpening path is mainly controlled by adaptive filters; contrast enhancement is needed in detail areas; little or no sharpening is needed in smooth areas. Literature [18] proposed a subimage histogram equalization enhancement method based on exposure, and the exposure threshold was used to divide the original image into subimages with different gray levels. Literature [19] proposed a hybrid image enhancement algorithm, which is based on the contourlet transform of sharp frequency localization and adaptive histogram equalization. Literature [20] reset the coefficients of the Laplacian pyramid directly after decomposing the image with the Laplacian pyramid, which is easier to operate on the coefficients, but it will cause a halo. The initial weights of BPNN (BP neural network) are optimized using an improved GA in literature [21]. The simulation results show that this method effectively improves the convergence speed of the BP neural network by reducing the likelihood of it falling into a local minimum. Literature [22] proposed the concept of self adaptation, which adaptively adjusts the crossover rate and mutation rate in response to individual sensitivity and population diversity change, thereby improving GA convergence speed. A better GA (Genetic Algorithm) based on population dissimilarity was proposed in literature [23]. The algorithm uses a heuristic crossover strategy and can adjust the crossover scale, mutation scale, and mutation rate of each gene in the mutant according to the population's diversity, avoiding premature population convergence and speeding up evolution.

\section{Research Method}

3.1. Demand Analysis of Digital Enhancement Technology in Sculpture Creation. Digital art is an artistic means by which artists use computer technology as a support to simulate three-dimensional scenes through digital information. This way, artists' creative consciousness is brought into space from the plane, and an impossible thing is realized, which greatly improves the scope of art. The Internet's high-speed media has enhanced the distance between the audience and technology, and the aesthetic interaction between the audience and art [24].

In the information age, computer technology endows art with infinite possibilities, which makes its creation and expression tend to be diversified. In the art of sculpture, with the intervention of $3 \mathrm{D}$ digital software technology, a sculpture is presented to everyone with a brand-new look. The demand for digital technology in sculpture creation is manifested in two aspects:

(1) In the ordinary sculpture creation practice, using computer to engrave $3 \mathrm{D}$ model, the structure of the model is analyzed clearly at any time by marking, and undo and advance continuously, which is a function that traditional sculpture cannot achieve. Practicing the component model in the software is helpful for everyone to understand and master the structure.

(2) In some large-scale sculpture creation, it is necessary to enlarge the model according to the manuscript. In the real world, there are some errors in the data of the scanning model, which cannot meet the precise requirements of large-scale projects. However, the data of the model carved in the software are very accurate, which can ensure the accuracy of the scaled size.

Sculpture molding is a time-consuming and difficult process in traditional sculpture creation. First and foremost, the sculpture image must be designed. A draft or a sample can be used in the design process. Sculpture mud, clay, oil mud, pulp, gypsum, and other easy-to-form materials are commonly used to create samples. The clay sculpture is then 
made in equal or reduced proportion to the draft or sample. Of course, there are times when the draft or sample is not used, and the equal proportion creation is performed on the spot. It is necessary to construct the sculpture skeleton as well as screen, and suppress the clay for a sculpture before beginning to create the clay sculpture. Figure 1 is a schematic diagram of the sculpture-making process under traditional technical conditions.

In the aspect of 3D digital molding technology, it is necessary to have a deep understanding of 3D printing, engraving technology, and other digital molding technologies. To understand different molding processes, different molding materials, and basic properties of materials in $3 \mathrm{D}$ printing technology, understanding basic knowledge of these can predict the effect of shaping the work. Because for 3D printing technology, different molding processes are suitable for different molding structures; for example, a stereolithography apparatus process is suitable for a complex mechanism and precision requirements. Today's mainstream design software generally updates its version once a year, and each update will increase its function. In order to better create art, attention is always paid to software version upgrades, and new features of the software are constantly studied.

When using 3D digital modeling technology to create sculptures, it is essential to master the basic knowledge of sculptures and basic techniques of creation, and it is also necessary to master 3D digital software and fully understand related molding technologies. Cultural literacy refers to the sculptor's understanding, accumulation, mastery, and perception of human cultural knowledge.

To begin with, only when sculptors have a thorough understanding of human culture, particularly art culture, the sculptures they create can have full vitality because they bear the connotation of human culture, and they can perform the aesthetic education function that art is supposed to perform. Traditional sculpture art is rooted in a rich historical and cultural heritage, as well as a rich national culture. It is more easily accepted and recognized by the audience because it has a strong affinity with the audience's psychology and feelings. As a result, cultural genetic defects of new means can only be compensated for when digital sculpture creators use their own cultural literacy and integrate cultural factors into sculpture creation.

Secondly, sculptors must have the ability to discover beauty. Artists should be discerning and discover the beauty that ordinary people cannot find so as to remind people that there are always beautiful things in the world. Furthermore, an artistic expression ability is an essential artistic accomplishment for artists. Art expression refers to the process of using professional skills to express artistic thoughts, that is, artistic creation.

In addition, as a sculptor uses modern technology to create a sculpture, it is very important to have the quality of science, especially computer science. Combining cultural literacy, artistic literacy, and scientific literacy and creating excellent digital sculpture works is one of the necessary qualities for sculptors who use 3D digital molding technology to create.

\subsection{Digital Enhancement Application of Traditional Sculpture} Art. GA (Genetic Algorithm) is a kind of randomized adaptive search algorithm that draws lessons from natural selection and natural genetic mechanism in biology. Because GA is a global optimization search algorithm, it can quickly and effectively calculate complex nonlinear multidimensional data space. Using a membership surface instead of a threshold surface can also realize adaptive dynamic image segmentation based on pixel clustering.

Several key steps in the implementation of GA based on the fuzzy membership surface are as follows [25]:

(1) Chromosome coding. Because the membership surface is used to realize dynamic image segmentation, chromosomes are encoded into a two-dimensional matrix with membership function values of each pixel as elements. The membership function value is a real number within $[0,1]$. For the convenience of operation, the membership function value of each pixel is quantized by 8 bits so that the solution space is $256^{M \times N}$.

(2) Initializing the population. Let the population size be $K$. Firstly, the gray range of the image is sampled at equal intervals, and $K$. thresholds are collected for global thresholding. Then, $K$ membership functional value surfaces $u_{2}(x, y ; T)$ about the background fuzzy set are calculated as follows:

$$
u_{2}(x, y ; T)= \begin{cases}\frac{1}{2}\left|\frac{f(x, y)-m_{1}(T)}{C}\right|^{\alpha}, & 0 \leq f(x, y) \leq T, \\ 1-\frac{1}{2}\left|\frac{f(x, y)-m_{2}(T)}{C}\right|^{\alpha}, & T \leq f(x, y) \leq L,\end{cases}
$$

where constant $C$ is the normalization factor, $T$ is any one of the $K$ thresholds, and $m_{1}, m_{2}$ is the average gray value of two types of pixels, which can be estimated from both sides of $T$ in histogram $h(g)$ :

$$
\begin{aligned}
& m_{1}(T)=\frac{\sum_{g=0}^{T} g h(g)}{\sum_{g=0}^{T} h(g)}, \\
& m_{2}(T)=\frac{\sum_{g=T+1}^{T} g h(g)}{\sum_{g=T+1}^{T} h(g)} .
\end{aligned}
$$

$\alpha$ is the compactness parameter of the mean value of the element pair within the class, which can describe fuzzy semantics such as approximate, close, and similar, $\alpha \geq 0$.

(3) Designing fitness function. The mask selects the following two energy functions: 




FIGURE 1: Sculpture-making process under traditional technical conditions.

$$
\begin{aligned}
& E_{1}=\sum_{x=1}^{M} \sum_{y=1}^{N} \sum_{l=-d}^{d} \sum_{k=-d}^{d}\left[u_{2}(x, y)-u_{2}(x+l, y+k)\right]^{2}, \\
& E_{2}=\sum_{x=1}^{M} \sum_{y=1}^{N} \sum_{l=-d}^{d} \sum_{k=-d}^{d}\left[u_{2}(x, y)+u_{2}(x+l, y+k)-1\right]^{2},
\end{aligned}
$$

where $E_{1}$ is a consistency measure, and the smaller $E_{1}$ is, the closer the membership values of adjacent pixels with respect to the same class. $E_{2}$ is a measure of mutual exclusion. The larger $E_{2}$ is, the greater the difference between the membership values of adjacent pixels with respect to different classes.

Horizontal filtering, vertical filtering, downsampling, upsampling, and difference calculation are all part of the Laplacian algorithm. Vertical filtering includes adding horizontal boundary padding and vertical filtering, whereas horizontal filtering includes adding vertical boundary padding and horizontal filtering. Each row's two padding elements are calculated from four elements of their neighboring peers, and the process is independent and irrelevant with good parallelism. After padding is applied, the $1 * 5$ calculation window is shifted backward one bit at a time, and the values of five elements in one row are used to calculate the target value corresponding to the window's central element in the target matrix. Each window size's element calculation is relatively independent, resulting in good parallelism.

As shown in Figure 2, the upsampling is divided into two parts: boundary calculation and body calculation. The boundary value is calculated from the values of its adjacent rows or columns. It moves backward one bit in turn with a window of $1 * 2$ size, gets two elements of one row at a time, and calculates the two-element values of the boundary row of the target matrix. Actually, the first element value is directly filled, and the second value is calculated from the two values obtained. If the number of rows is odd, it is necessary to calculate the boundary value of the first row, and the rest of the row can be filled with the main body.

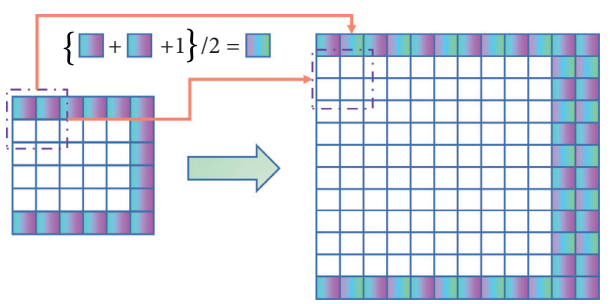

FIGURE 2: Upsampling process.

If the number of columns is odd, only the value of the last column needs to be calculated, and the rest is filled by the main part. Subject: moving back one bit by row and column in a window of $2 * 2$ sizes, obtaining four elements of the source matrix each time, and calculating the values of four elements of the target matrix.

The model coordinate system and image projection in the virtual space are transformed into the geometric transformation of the camera coordinate system. Camera pose estimation is to calculate the pose of the mobile phone camera in real time, and the initial matching point set is obtained after obtaining the feature matching of the abovementioned image and the identification image.

With homography matrix transformation, the reversible transformation from real projective plane to projective plane, this mapping can be expressed by matrix multiplication:

$$
P=A \times W \times M,
$$

where $P$ represents the coordinates of the two-dimensional image; $A$ represents the camera parameter matrix; $W$ stores the camera's internal parameters; $M$ is the coordinate of the three-dimensional world.

$W$ consists of two parts: the physical transformation of the object plane for positioning observation and the projection $R, T$ of the parameter matrix in the current camera. $[R \mid T]$ is a $3 \times 3$ matrix, and the world coordinate system is transformed into the camera coordinate system. The corresponding projection transformation relation is as follows:

$$
W=[R \mid T] .
$$

The world coordinate system and camera coordinate system used by the Unity3D rendering engine are both lefthanded coordinate systems. On the android platform, JAVA and C\# methods are called each other through Android JDK, and the camera pose parameters of the parameter matrix $R$ and $T$ are transferred from Android to Unity3D so as to realize the tracking registration of the virtual model from $2 \mathrm{D}$ to $3 \mathrm{D}$.

In this way, the algorithm as a whole will have a conditional judgment for one row, which contains a conditional compilation for the last column parity judgment; the last line contains a conditional compilation judgment, which contains a conditional compilation for the last column of parity judgment; partial calculation includes a conditional compilation of parity judgment for the last column. 
SIMD (single instruction multiple data) is an instruction to operate multiple data. It is an extension of the basic instruction set of the CPU. It is mainly used to provide finegrain parallelism, that is, the parallel operation of small pieces of data.

In view of the simplicity of the Laplacian algorithm here, this part is combined with vertical filtering, which reduces the calculation of some data. While optimizing SIMD of vertical filtering, the data needed for downsampling are directly reserved. There are a lot of shift operations in the improved algorithm, all of which use a shuffle instruction to align data. The upsampling algorithm is not suitable for SIMD optimization because of its many judgment conditions.

\section{Result Analysis and Discussion}

Traditional sculpture is taken as an example for developing a traditional sculpture image recognition system. the mobile device's camera is initialized, keyframe photos of the real scene are obtained, feature points are matched to identify feature images, the mobile terminal's pose is estimated, the spatial distance is calculated between the virtual model and the real scene, and superimposed on the current window to generate the virtual model's fused output. Figure 3 shows a comparison of the effects of various feature detection algorithms.

Other methods are likely to have a large number of features, slow calculation speeds, and a limited number of feature extraction speeds. The algorithm described in this paper has a faster calculation speed and a higher recognition efficiency of feature points, making it suitable for displaying complex textures of traditional sculptures on mobile devices. The algorithm detects local features with a high recognition rate and a high tolerance for light, noise, and small visual angle changes. Furthermore, this algorithm's feature distribution has more correct areas, and the matching accuracy is relatively high. The improved feature matching algorithm of this algorithm performs better in real time and highefficiency systems.

In the image segmentation method based on basic GA, nine traditional sculpture images of $191 \times 257$ are segmented by setting different segmentation parameters. Figure 4 shows the corresponding relationship between parameters and images.

As can be seen from Figure 4, the size of the cluster has a great influence on the segmentation effect and the computer time consumed by image segmentation. The larger the population, the better the segmentation effect, and of course, the more computer time it takes. Search algebra also has a great influence on the segmentation effect. The more search algebra, the better the segmentation effect. However, the search algebra has little influence on the computer time consumed by segmentation; so in order to improve the segmentation effect without consuming too much computational time, the search algebra can be increased. The number of variations has little effect on the segmentation effect, and it also has little effect on the computer time of image segmentation.

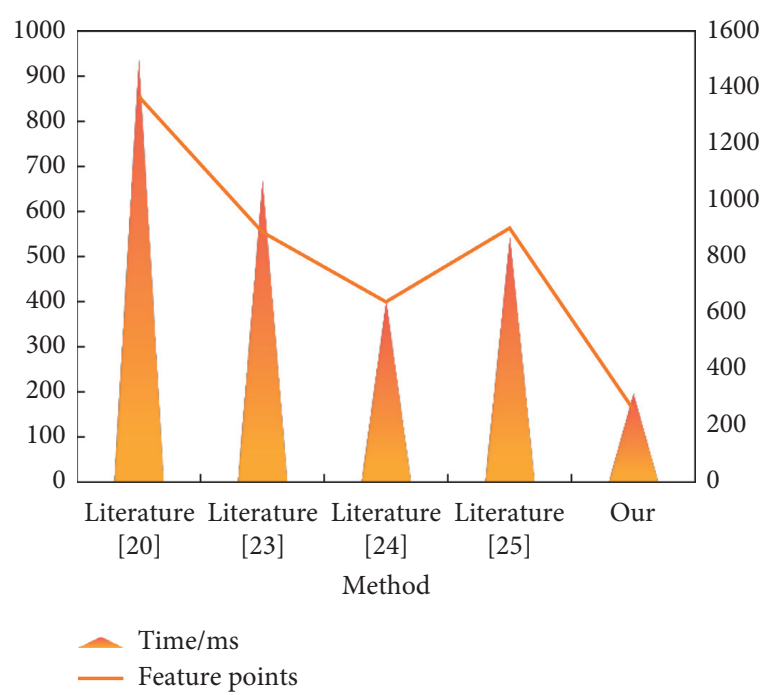

FIGURE 3: Effect comparison of different feature detection algorithms.

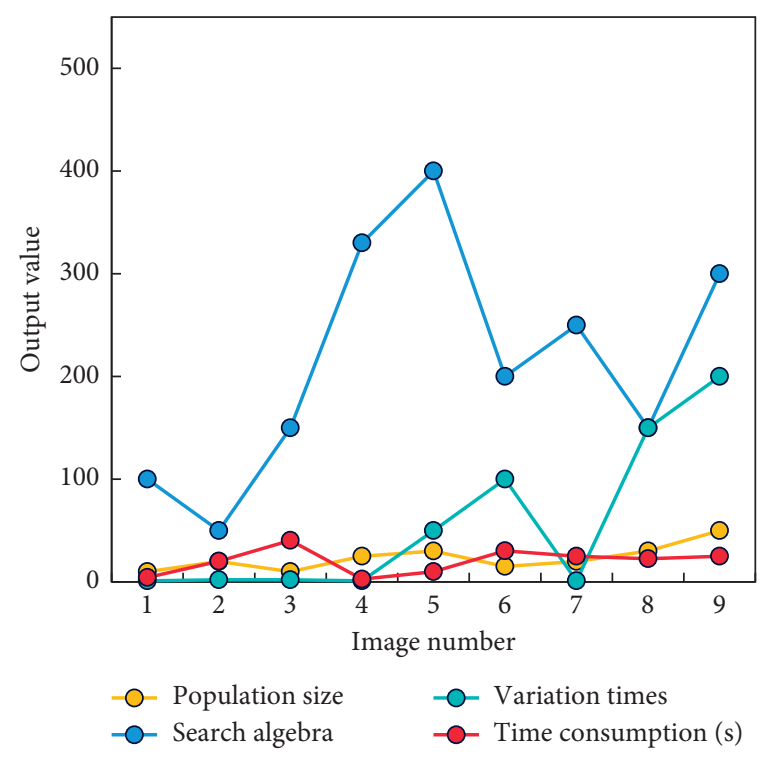

FIgURE 4: Segmentation result.

The adjustable parameters of the GA segmentation method based on the fuzzy membership surface include population size, mutation times, search algebra, and fitness function parameters. Next, by setting different parameters, the image is segmented, and the segmentation results are compared. The corresponding relationship between parameters and images is shown in Figure 5.

It can be seen from Figure 5 that the size of the population has a great influence on the segmentation effect and the computer time spent on image segmentation. The larger the population, the better the segmentation effect, and of course, the more computer time it takes. Search algebra also has a great influence on the segmentation effect. The more search algebra, the better the segmentation effect. However, the search algebra has little influence on the computer time consumed by segmentation; so in order to improve the 


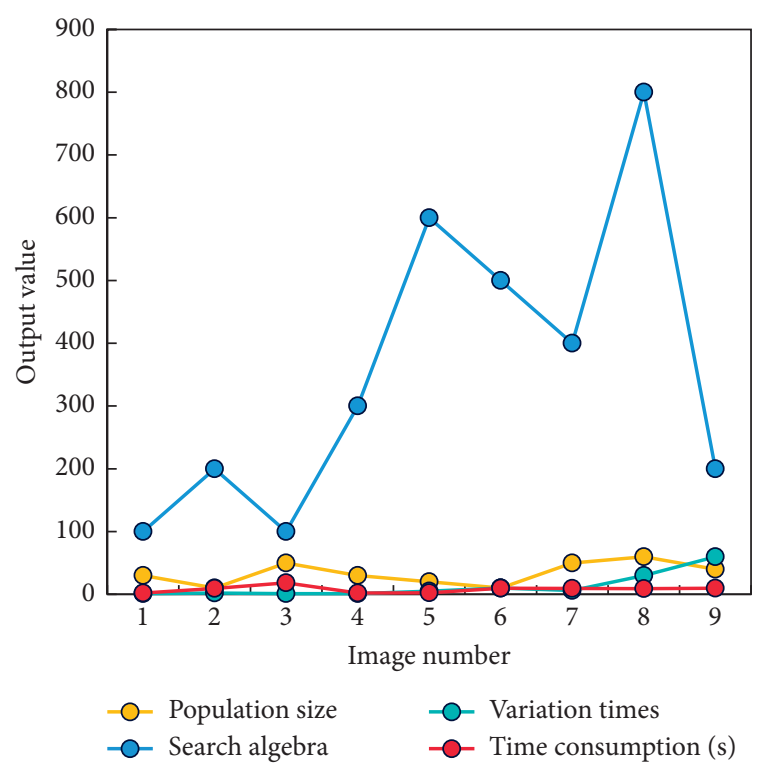

Figure 5: Segmentation result.

segmentation effect without consuming too much computational time, the search algebra is added. The number of variations has little effect on the segmentation effect, and it also has little effect on the computer time of image segmentation.

The Figure 6 shows the performance comparison among the CPU (central processing unit) version, SIMD optimized version, basic GPU (graphics processing unit) version, and optimized GPU version of the Laplacian algorithm.

It can be seen from Figure 6 that as the image size continues to grow, the acceleration ratio also increases. Therefore, it can be seen that using the SIMD optimization technique to optimize the CPU serial code is significant for improving algorithm performance, and it is a very effective optimization method.

The performance comparison between the optimized version of GPU and the basic version of GPU will be compared in detail in the performance improvement chart brought by the following optimization methods. Here, in order to show the acceleration of GPU relative to the CPU version, the performance improvement of the optimized version of GPU for the basic version of GPU is not obvious.

The main feature of geometric sculpture is that its modeling is mainly geometric figures. In $3 \mathrm{D}$ software, it is the modeling obtained by adding or subtracting figures through the Boolean operation. Although geometric sculpture is not as rich in surface changes as other shapes, it gives people a simple and straightforward feeling with its unique and minimalist shape.

Structural sculpture mainly emphasizes the structure of works, which is its external manifestation and also the embodiment of its essence and spirit. Usually, the structure is not only the support but also the main component of the enriching sculpture form, and its change will directly affect the change of the sculpture shape. In many art exhibitions, you can see many structural sculptures with rich changes and aesthetic feelings.

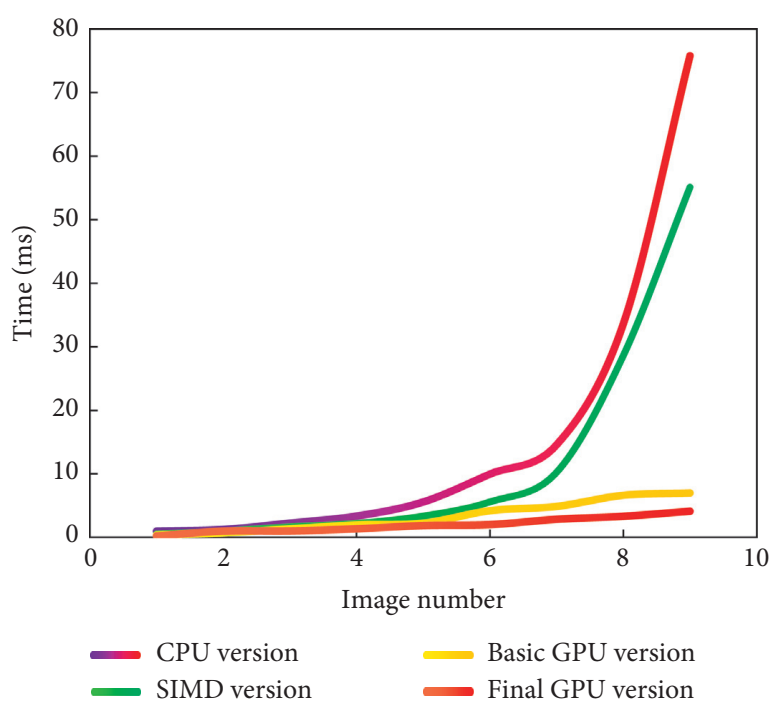

Figure 6: Performance comparison of optimized GPU versions.

The boundary padding part is integrated with the horizontal filter kernel, and the horizontal filter kernel is optimized by combining boundary optimization, conditional compilation, and vectorization. The performance results are shown in Figure 7.

As can be seen from Figure 7, with the increase of the image size, the acceleration ratio greatly increases, and the acceleration ratio is between 14 and 173 .

An improvisation is a form of entertainment for artists, as it allows them to express their innermost feelings as quickly as possible. It is a dialogue between mind and matter, as well as a state of selflessness, that is embodied in sculpture creation. The characteristics of the object are conformed and caught, the "truth" of the object lurking in the depths are dig out, and finally, the work is completed according to the original characteristics of creative materials. This method of creation necessitates the author's artistic ability, as well as keen insight and judgment, and the process of creation is the process of conforming to "God's will" and gradually releasing it from mixed purity. Because the scheme is uncertain, each step is the work's birth process, and each step gives the creator a new feeling.

Figure 8 shows the performance improvement of the upsampling algorithm after kernel fusion, conditional compilation, and boundary processing optimization. It can be clearly seen that with the increase of the image size, the speedup ratio increases greatly.

The choice of technical means will undoubtedly affect the presentation form of sculpture works, as sculpture is an artistic form expressed by modeling features. We can only deal with the sculpture language and then express artistic ideas using certain technologies. Many sculpture creators nowadays use the pursuit of pure art as an excuse to avoid personally participating in the technical work of sculpture, believing that as long as the artistic conception is presented by themselves, it is regarded as their own creation, regardless of who provides the technical work or what kind of technology is used to complete it. As a result, once technological 


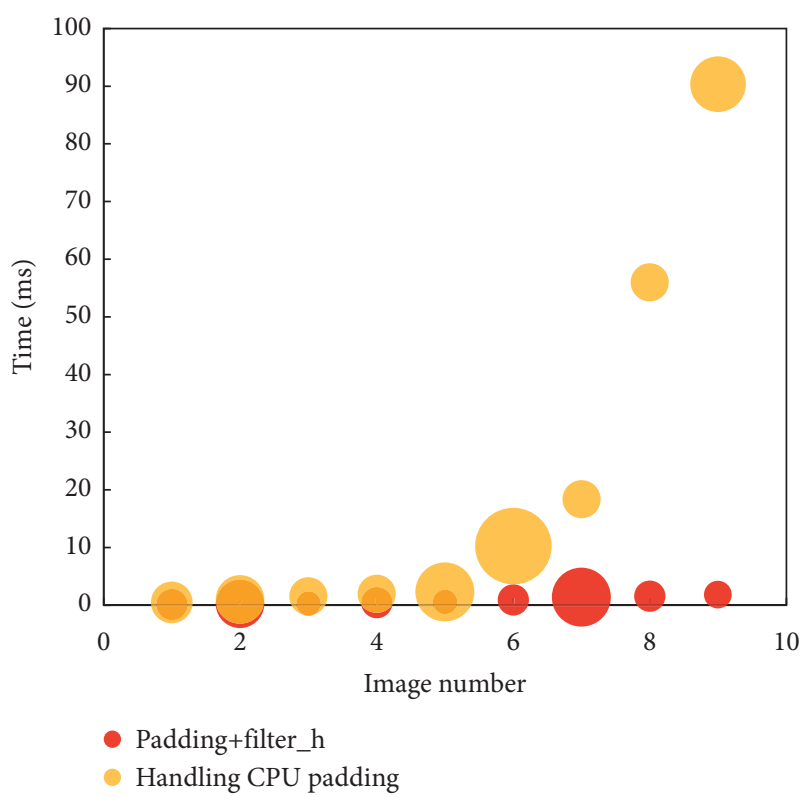

Figure 7: Performance comparison of boundary padding and horizontal filter kernel before and after optimization.



FIGURE 8: Upsampling optimization performance comparison.

research has progressed to a certain point, digital sculpture creation requires the guidance of artistic theory and artistic achievement, as well as the coordinated development of technical means and artistic achievement so that it can not only demonstrate technology but also create artistic works that shock the soul.

Figure 9 shows the performance of several optimization methods with obvious performance improvement for the basic GPU version. This section gives the performance comparison data of vertical filtering and downsampling after partial optimization.

From Figure 9, it can be seen that vertical filtering and downsampling are used to optimize by processing four columns at the same time, which can improve the distinguishable performance. It can be seen that the simplified

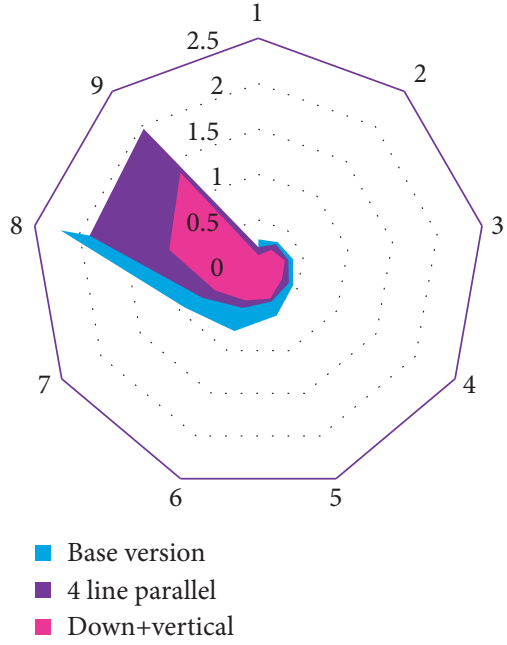

FIGURE 9: Performance improvement brought by the optimization method on GPU.

algorithm is indeed helpful to performance optimization, but it is not the bottleneck problem of the algorithm performance and has limitations on the optimization effect of the algorithm. Adding the optimization method of padding does not improve the performance, but it does enable the GPU kernel to handle input images with more diversified sizes, and the performance will not be reduced by adding conditional judgment, which theoretically optimizes the algorithm in the process of improving the algorithm.

Digital modeling technology participates in sculpture design and production. Although it has brought a more efficient way of creation to sculpture art and some negative effects to traditional sculpture art, as long as the new technology is treated calmly and used, the development of sculpture art will be promoted.

Some people who have not received professional sculpture training can also use software to make sculpturelike shapes, but such shapes lack the language features that sculpture should possess and reflect through professional knowledge. After all, a pile of mud made by people without professional knowledge in sculpture art cannot be called "mud." The sculpture is called art because it has thresholds and requirements.

\section{Conclusion}

Three-dimensional technology provides a new carrier for sculpture creation, and sculpture works are displayed in electronic display instruments through electronic information technology, which just promotes the development and spread of sculpture culture and the development and progress of the whole sculpture creation industry. Software belongs to software thinking. Therefore, in artistic creation activities, the creator's thinking is the main guide, supplemented by software thinking. Dealing with the interaction between the two will make artistic creation more efficient, provide artists with modeling forms beyond their own thinking limits, bring new thinking enlightenment, and stimulate artists' creative ability. This paper discusses the 
method of GA in image restoration processing, and the experimental results show that the image restoration processing method based on GA can improve the image resolution and quality. Future works will continue to solve the problem of matching recognition between traditional sculpture art and the human face. By recognizing facial expressions, we can display different mask expressions with rich cultural connotations and further improve the mobile terminal to add new functions in displaying other interactive experiences of traditional sculpture art.

\section{Data Availability}

The data used to support the findings of this study are included within the article.

\section{Conflicts of Interest}

The author declares that he has no conflicts of interest.

\section{Acknowledgments}

This study was supported by Key Project: Provincial Key Quality Process Project of Institutions of Higher learning in Anhui Province in 2019 by the Department of Education: Research on the Teaching Reform of Sculpture Course in Art Design Major in Colleges and Universities--A Case study of Environmental Art Design Major; Major Projects: 2019 Anhui University Humanities and Social Science Research Major Projects: A study on the poetic creation of Chinese sculpture and space environment in the new era; Key Project: Special research project for postgraduate tutors of Hefei Normal University in 2021 Research on Practice Training and Ability Cultivation of Postgraduates in Jade Teaching (DSKY10); and General project: school level quality engineering general project of Anhui University of architecture and architecture in 2021 Reform and practice of sculpture teaching system of environmental design specialty in Architectural Colleges and Universities (2021jy71).

\section{References}

[1] B. Pitman, "Pharma art-abstract medication in the work of beverly fishman," Jama the Journal of the American Medical Association, vol. 319, no. 4, p. 326, 2018.

[2] M. Vicente, "Images of people at work: the videomaking of Darcey Lange," Humanities and Social Sciences, vol. 24, no. 10, pp. 1577-1600, 2017.

[3] D.-H. Lee, "Standard for protection of copyright for scale models of real buildings," The Jounal of Comparative Private Law, vol. 24, no. 3, pp. 1447-1480, 2017.

[4] C. Silva, G. Vélez, and H. A. Colorado, "Patina in the construction of the poetic bronze image: S," Heritage Science, vol. 5, no. 1, p. 36, 2017.

[5] L. Ivimey, "Crispin II," Textile Fibre Forum, vol. 12, no. 127, pp. 36-37, 2017.

[6] H. Martin, "James shaw," Architectural Digest, vol. 76, no. 6, p. 26, 2019.

[7] V. Croci, "Heatherwick studio," Domus, vol. 4, p. 56, 2019.

[8] G. Comoglio, "Annette gigon/mike guyer architekten," Domus, vol. 2, p. 19, 2019.
[9] W. Cai, D. Liu, X. Ning, C. Wang, and G. Xie, "Voxel-based three-view hybrid parallel network for $3 \mathrm{D}$ object classification," Displays, vol. 69, p. 102076, 2021.

[10] H. Pearman, "Americans abroad," Architectural Record, vol. 206, no. 1, pp. 50-54, 2018.

[11] H. Xin, K. Zhang, H. He, W. Hu, and M. Zhang, "Fidelity enhancement in high-data-rate digital mobile fronthaul with sample bits interleaving and unequally-spaced PAM4," Optics Express, vol. 25, no. 5, pp. 5559-5570, 2017.

[12] A. Covaci, G. Ghinea, and C. H. Lin, "Multisensory gamesbased learning-lessons learnt from olfactory enhancement of a digital board game," Multimedia Tools and Applications, vol. 77, no. 16, pp. 1-19, 2018.

[13] L. Wen, H. Qi, and S. Lyu, "Contrast enhancement estimation for digital image forensics," ACM Transactions on Multimedia Computing, Communications, and Applications, vol. 14, no. 2, pp. 49.1-49.21, 2018.

[14] W. Wang, H. Li, P. Zhao et al., "Advanced digital signal processing for reach extension and performance enhancement of 112 gbps and beyond direct detected DML-based transmission," Journal of Lightwave Technology, vol. 37, no. 1, pp. 163-169, 2019.

[15] L. Feng, X. Mu, and C. Lin, "Adaptive digitization and variable channel coding for enhancement of compressed digital mobile fronthaul in PAM-4 optical links," Journal of Lightwave Technology, vol. 1, no. 21, 2017.

[16] J. C. Russ and R. P. Woods, "The image processing handbook, 2nd ed." Computers in Physics, vol. 8, no. 6, p. 177, 2017.

[17] X. Wang, S. Ghosh, and S. W. Guo, "Quantitative quality control in microarray image processing and data acquisition," Nucleic Acids Research, vol. 29, no. 15, pp. E75-5, 2001.

[18] I. R. Kamel, C. Georgiades, and E. ., K. Fishman, "Incremental value of advanced image processing of multislice computed tomography data in the evaluation of hypervascular liver lesions." Journal of Computer Assisted Tomography, vol. 27, no. 4, pp. 652-656, 2017.

[19] K. Aoki, "Server for implementing image processing functions requested by a printing device," Environmental Pollution, vol. 152, no. 3, pp. 543-552, 2018.

[20] G. Wu, B. Masia, A. Jarabo et al., "Light field image processing: An overview," IEEE Journal of Selected Topics in Signal Processing, vol. 11, no. 7, pp. 926-954, 2017.

[21] G. Cheung, E. Magli, Y. Tanaka, and M. K. Ng, "Graph spectral image processing," Proceedings of the IEEE, vol. 106, no. 5, pp. 907-930, 2018.

[22] S. H. Kang and J. Shen, "Quantum TV and applications in image processing," Inverse Problems and Imaging, vol. 1, no. 3, pp. 557-575, 2017.

[23] N. Yagi, "Special issue image processing. 3. New application of image processing. 3-1 image processing in broadcasting.:3-1 image processing in broadcasting," Terebijon Gakkaishi, vol. 46, no. 11, pp. 1439-1442, 2017.

[24] K. Senthilkumar, N. K. Vivek, and E. Vijayan, “An efficient image processing method based on web services for mobile devices," IOP Conference Series: Materials Science and Engineering, vol. 263, no. 4, Article ID 042023, 2017.

[25] P. Dala-Krishna, "Ultrasound image processing to render three-dimensional images from two-dimensional images," Acoustical Society of America Journal, vol. 131, no. 5, Article ID 4231, 2017. 